\title{
RENTA BÁSICA E INCENTIVOS LABORALES: UNA APROXIMACIÓN DESDE LA TEORÍA DE JUEGOS*
}

\author{
Basic Income and Labour Incentives: A Game Theory Approach
}

\author{
JORDI TENA-SÁNCHEZ** \\ Universitat Autònoma de Barcelona \\ JOSÉ A. NOGUERA \\ Universitat Autònoma de Barcelona
}

\begin{abstract}
RESUMEN
En el presente trabajo se discute cuál puede ser el impacto de los incentivos laborales de la introducción de una Renta Básica de Ciudadanía. Más en concreto, trata de explorarse si dicha medida está condenada al fracaso debido a que produciría una evacuación masiva del mercado de trabajo o si, por el contrario, tal evacuación no constituye un escenario plausible y, por tanto, la propuesta resulta sostenible y viable en este aspecto. Para llevar a cabo dicha discusión se emplean modelos formales de teoría de juegos, ya que estos presentan diversas ventajas frente a la elaboración mental intuitiva. El trabajo concluye que, como sostienen los defensores de la Renta Básica, la medida resulta viable y sostenible en los escenarios empíricamente más plausibles.
\end{abstract}

Palabras clave: Políticas sociales, Estado del Bienestar, workfarismo, disposiciones hacia el empleo, modelos formales.

\begin{abstract}
This paper discusses the likely impact on job incentives of the introduction of a Citizens' Basic Income. We try to explore whether this proposal would fail because it would cause a massive retreat from the labour market or, on the contrary, this is not a plausible scenario and therefore the proposal is feasible and sustainable in this respect. To this aim, we use formal game theoretical models, since they have several advantages compared with simple intuitive mental speculation. The paper concludes that, as Basic Income supporters argue, the proposal is feasible and sustainable in the most empirically plausible scenarios.
\end{abstract}

Key words: Social policies, welfare state, workfare, job dispositions, formal models.

* Este trabajo se ha desarrollado en el marco de un Proyecto I+D+i con referencia CSO2012-31401 y de un proyecto CONSOLIDER-INGENIO con referencia CSD 2010-00034, financiados respectivamente por el Ministerio de Ciencia e Innovación (MICINN) y por el Ministerio de Economía y Competitividad.

** Los autores muestran su agradecimiento a Laia París, cuya tarea como estudiante en práctica en el Grupo de Sociología Analítica y Diseño Institucional (GSADI) fue de gran utilidad en las fases iniciales de este trabajo. 


\section{INTRODUCCIÓN}

En el presente trabajo se asume la definición "estándar" de Renta Básica (en adelante $\mathrm{RB})$, a saber: la RB es una prestación monetaria, financiada mediante impuestos, que la administración paga de forma periódica (por ejemplo, mensualmente) a cada ciudadano. Se trata de una prestación que se paga a los individuos (no a las familias o a los hogares) de forma incondicional (es decir, sin tomar en consideración la edad o el nivel de renta del individuo, que este haya cotizado previamente, que tenga o no un empleo, o cualquier otra consideración) e independientemente de cualquier otra renta que el individuo pueda percibir. ${ }^{1}$ En lo que se refiere a la cuantía de la prestación, esta debe ser la máxima posible que resulte sostenible en el tiempo (Van Parijs, 1997).2

Una de las cuestiones acerca de las que más se ha especulado en el debate de la RB es la de cómo afectaría a los incentivos laborales. Es cierto que el efecto de las prestaciones monetarias en los incentivos laborales de los individuos es discutido para cualquier tipo de prestación, pero la incondicionalidad de la RB hace la cuestión si cabe más perentoria. La discusión en la teoría social normativa respecto de la explotación y el parasitismo a los que la RB podría dar lugar ha sido muy prolija, ${ }^{3}$ pero no así la literatura científico-social de cómo determinar en qué medida esos escenarios catastrofistas son o no plausibles.

Existen diferentes estrategias, todas ellas complementarias, que los científicos sociales interesados en la cuestión podrían adoptar. Estas estrategias podrían consistir en el estudio de casos reales de aplicación de una $\mathrm{RB}^{4} \mathrm{o}$ de las actitudes y motivaciones empíricas hacia el trabajo y el empleo, en la realización de experimentos sociales, ya sean de campo o de laboratorio, ${ }^{5}$ en la construcción de modelos de simulación multiagente, etc. Un tipo particular de estrategia, en el marco donde se sitúa este trabajo, consiste en la elaboración de modelos teóricos que permitan un análisis sistemático de la situación y

1 Esta es la definición adoptada, por ejemplo, por Van Parijs (1997) o por la Basic Income Earth Network (BIEN): http://www.basicincome.org. Puede verse una panorámica de las discusiones de la propuesta de la Renta Básica en Widerquist et al. (2013).

2 Una cuestión adicional es la de la relación de la RB con otras prestaciones y servicios sociales. Algunos defensores de la medida, destacadamente determinados economistas neoliberales y filósofos políticos libertarianos, como Milton Friedman, Friedrich Hayek o Hillel Steiner (1992), han propuesto que la RB debería substituir al resto de prestaciones del Estado de Bienestar. No obstante, para la mayoría de defensores de la medida (ver nota anterior), como principio general, la RB debe añadirse al resto de prestaciones y servicios existentes, no substituirlos. No obstante, también para estos autores e instituciones, la introducción de una RB debería suponer la eliminación de todas las prestaciones mínimas que caigan por debajo de la cuantía de la RB (incluidos los gastos fiscales como el mínimo vital del IRPF y otras muchas exenciones y deducciones). Por tanto, aunque evidentemente la medida tendría un coste neto considerable, este sería mucho menor del que a primera vista pudiese parecer.

3 Van Parijs (1997) y Elster (1986), respectivamente a favor y en contra de la medida, constituyen dos de las referencias más relevantes en este debate.

4 No obstante, si bien existen algunas experiencias que pueden permitir aproximaciones empíricas al fenómeno, en la actualidad no existe ninguna RB como la que se define. El caso más cercano es el Alaska Permanent Fund. Sin embargo, este caso no es de aplicación aquí, ya que dicho dividendo que no se financia con impuestos sino con un fondo obtenido sobre la explotación de recursos naturales.

5 Véase Noguera y Wispelaere (2006) para una discusión de las posibilidades del segundo método, y Widerquist (2005) para una discusión de los resultados de algunos ejemplos clásicos del primero. 
de sus implicaciones lógicas y teóricas. Los modelos formales presentan algunas ventajas frente a la mera elaboración mental intuitiva. La modelización obliga al investigador a hacer explícitos sus supuestos (que de otro modo podrían permanecer ocultos, incluso para él mismo), permite la calibración empírica de los mismos, así como la derivación de implicaciones lógicas fértiles para la investigación.

En el terreno de los incentivos laborales, si bien los modelos económicos de oferta de trabajo son numerosos, lo más habitual es que estos sean de tipo paramétrico, a saber, se supone que los trabajadores son individuos aislados que reaccionan a un determinado input en el mercado laboral (la introducción de un determinado incentivo/desincentivo laboral como puede ser una RB) sin tener en cuenta las decisiones de los demás y sin que sus acciones afecten a la conducta de estos. El presente trabajo se plantea ir un paso más allá y emplear la caja de herramientas de la teoría de juegos de estrategia para elaborar modelos teóricos que permitan la exploración sistemática de la situación teniendo en cuenta las dinámicas de interacción entre los agentes. El valor de los modelos de juegos es principalmente conceptual, como argumenta Elster (2007: 312), estos permiten iluminar la estructura de la interacción social. Las consecuencias que dicha estructura comporta pueden no ser visibles de inmediato. Al obligarnos a precisar la naturaleza de dicha estructura, la teoría de juegos puede volver esas consecuencias manifiestas. La teoría de los juegos de estrategia puede ser una buena herramienta formal para los objetivos de este trabajo, porque con una RB financiada con impuestos sobre los salarios y el capital, e indexada con la evolución de los mismos (por ejemplo, con el PIB per cápita), tendremos un escenario de interdependencia estratégica como aquellos a los que se aplica típicamente dicha teoría.

Permítasenos insistir en que nuestro análisis se centrará en cuestiones de tipo teórico y factual, no en discusiones normativas. Es posible distinguir dos tesis diferentes en las críticas más habituales contra la RB: si se introduce una RB se producirá una evacuación masiva del mercado de trabajo (una predicción teórica y factual), y esto comportaría la explotación de los laboriosos por parte de los holgazanes, cosa que sería injusta (tesis normativa). En este trabajo pretendemos centrarnos en la primera tesis y dejamos de lado la segunda. Nótese que ambas tesis son independientes desde un punto de vista lógico: rechazar la predicción de hecho no nos obliga a rechazar el argumento normativo (podríamos aún sostener que aunque solo existiese un único holgazán explotador, eso sería ya injusto). De forma similar, rechazar la tesis normativa no implica necesariamente rechazar la predicción factual: podríamos considerar que la RB sería una medida justa, pero dudar de su viabilidad política y económica y, por tanto, de cómo podría llegar a afectar a los incentivos laborales.

Por otro lado, es necesario hacer explícito que en este trabajo tampoco estamos principalmente interesados en argumentos empíricos del tipo "una evacuación masiva del mercado de trabajo no sucedería en el mundo real". Estamos sobre todo interesados en un argumento conceptual: las críticas contra la RB se basan de manera directa en algunos supuestos acerca de las características de la naturaleza estratégica de la interacción en un mercado de trabajo con una RB, mientras que descartan otros. Así pues, esas críticas resultan sesgadas y descansan en opciones teóricas que no se exponen ni justifican de 
manera explícita. Dicho esto, es evidente que algunos de los escenarios que se explorarán resultan desde el punto de vista empírico más plausibles que otros, cosa que iremos señalando durante la discusión. Pero es menester tener presente que nuestro principal objetivo es emplear la teoría de juegos para explorar de modo sistemático las dinámicas de interacción en el mercado de trabajo a las que potencialmente se enfrentarían los agentes si se implantase una RB.

El resto del trabajo se organiza como sigue. En el segundo apartado presenta de manera formal el tipo de problema de acción colectiva al que da lugar la RB. A continuación se exponen los supuestos del modelo teórico. En el cuarto apartado se presentan y discuten los escenarios de interacción estratégica elaborados. Finalmente, el trabajo se cierra con un apartado de conclusiones en el que se discuten los resultados obtenidos.

\section{LA RENTA BÁSICA COMO PROBLEMA DE ACCIÓN COLECTIVA}

En lo que concierne a la relación entre la RB y los impuestos que la sufragan, existen diferentes escenarios posibles en función de la variabilidad o no de ambos elementos. En el presente trabajo se explorará el escenario más realista desde el punto de vista de la viabilidad política, que es aquel en que la RB es de una cuantía variable mientras que los impuestos son fijos. Así pues, cuantos más individuos trabajen y paguen impuestos, mayor será la cuantía de la RB y viceversa.

La existencia de una RB como la definida constituye un bien público impuro. Como es sabido, los bienes públicos se definen a partir de dos características básicas: no exclusividad y no rivalidad. La primera característica consiste en que, una vez producido el bien, no es posible excluir a nadie de su consumo, incluso a aquellos que no han contribuido a su producción. ${ }^{6}$ Por su parte, la segunda característica consiste en que el hecho de que un individuo consuma el bien no reduce su disponibilidad para otros ni aumenta los costes de producción.

La RB alberga claramente la primera característica, ya que por definición es universal. No obstante, alberga solo parcialmente la segunda, de ahí que no podamos definirla como un bien público puro. El hecho de que un nuevo individuo pase a cobrar la RB hace que disminuya la cuantía disponible para los demás. La provisión de la RB supone un problema de acción colectiva, porque, como con cualquier otro bien público, un agente racional puede estar tentado a beneficiarse del bien sin contribuir a su producción. No obstante, ciertamente el caso analizado presenta algunas características especiales que lo distinguen de los problemas habituales de provisión de bienes públicos. El salario (o el beneficio empresarial) que se obtiene fruto de la participación laboral, así como la autorrealización y el prestigio social asociados a la misma en nuestras sociedades, constituyen cuantiosos incentivos selectivos (materiales y morales) para la participación en la promoción del bien público que no suelen darse (al menos en esas dimensiones) en otras acciones colectivas. Este hecho explica que, como se verá en el siguiente apartado, el mantenimiento de la RB no dé lugar exactamente a los cinco juegos de la taxonomía 
clásica de Heckathorn (1996). ${ }^{7}$ Antes de entrar en la modelización de la situación se presentarán los supuestos básicos del modelo.

\section{SUPUESTOS DEL MODELO}

A continuación se explicitan y discuten (brevemente) los supuestos básicos del modelo:

- Los agentes disponen de dos estrategias posibles (trabajar -T-, no trabajar -NT-). Por razones de simplicidad, se prescinde de la cantidad y calidad del trabajo y se supone que todo el mundo que trabaja lo hace el mismo tiempo y con la misma productividad $y$, consecuentemente, gana el mismo salario y paga los mismos impuestos. Además, por trabajo se entenderá, exclusivamente, el trabajo remunerado. El trabajo no remunerado, el voluntariado, trabajo doméstico, etc., son irrelevantes aquí porque no producen renta.

- Existen dos clases generales de agentes: laboriosos, que prefieren trabajar aunque ya dispongan de una renta suficiente, y holgazanes, que no quieren trabajar si ya disponen de dicha renta y prefieren dedicarse a otras actividades. ${ }^{8}$ Se deja aquí de lado la cuestión de cuál sea el origen de esa preferencia, que se toma como dada o exógena. No obstante, obviamente la variedad de motivaciones en las que se asiente una determinada disposición hacia el trabajo puede ser muy amplio, pudiendo ir desde los (egoístas) deseos de acumular más renta o autorrealizarse, hasta consideraciones de tipo moral respecto de lo que constituye el deber propio, pasando por un largo etcétera. ${ }^{9}$

- Los juegos son de N jugadores (ego y todos los demás), la utilidad es ordinal y las jugadas simultáneas. ${ }^{10}$

- El mercado de trabajo está en equilibrio y no existen empleo ni desempleo involuntarios. Adicionalmente, puede haber individuos con la existencia asegurada al margen de la RB y del mercado de trabajo (aunque no se supone nada de niveles de subsistencia básica).

7 Los citados juegos son: Dilema del Prisionero, Dilema del Gallina, Dilema (o Juego) de la Seguridad, Dilema del Altruista y Juego del Privilegiado.

8 Aquí se toman prestados a los personajes de la parábola de Crazy y Lazy de Van Parijs (1997), y se analiza su interacción desde el punto de vista de la teoría de juegos.

9 Uno de los factores que pueden afectar a la predisposición de un individuo hacia el trabajo son las características de dicho trabajo. Así, que un individuo tenga un orden de preferencias de holgazán puede deberse a que solo puede optar a empleos alienantes para él, mientras que su orden de preferencias sería distinto si tuviese la oportunidad de conseguir un empleo creativo, en buenas condiciones, etc. Así, aunque, como hemos dicho, por simplicidad supondremos que todos los trabajos son iguales, el hecho de que los individuos alberguen disposiciones distintas hacia el trabajo nos permite capturar (al menos parcialmente) que los empleos difieren en cosas como las condiciones de trabajo, su potencial de autorrealización para quién los realiza, etc., y que esas cosas afectan a la disposición de los individuos hacia el trabajo.

10 Quizá una alternativa razonable sería modelizar los escenarios con juegos secuenciales. Al fin y al cabo, los mercados de trabajo no se crean de cero, sino que cada nuevo trabajador se incorpora a un mercado en el que ya hay agentes trabajando. En general, los resultados no cambian cuando se emplea un tipo u otro de juego. Se discutirán brevemente los tres casos en los que sí que se producen cambios. 
- Los individuos intentan maximizar dos cosas: sus preferencias por el trabajo/no trabajo y su renta global, que incluye la RB. En este sentido, los agentes tratan de resolver un trade-off entre renta y ocio.

\section{ESCENARIOS ESTRATÉGICOS}

\section{Tipos de agentes}

Antes de discutir las interacciones se presentarán con algo más de detalle los tipos de agentes que tomarán parte en las mismas. Los agentes laboriosos y holgazanes a los que se ha hecho referencia más arriba darán lugar cada uno a dos perfiles de agentes distintos en función del grado de incondicionalidad de su laboriosidad u holgazanería. De este modo tenemos: laboriosos incondicionales, laboriosos condicionales, holgazanes condicionales y holgazanes incondicionales.

\section{Laborioso incondicional (LI)}

El laborioso incondicional es un agente con una marcada predisposición hacia el trabajo. El Cuadro 1 muestra su orden de preferencias. Como se puede observar, tiene una estrategia dominante $\mathrm{T}$, de modo que preferirá trabajar en cualquier circunstancia, independientemente del número de free-riders en la población, de la tasa impositiva, así como de que perciba una $\mathrm{RB}$ o disponga de cualquier otra fuente de renta. Su última preferencia se refiere a aquel escenario en que nadie trabaja (NT/NT), ya que en un contexto así no recibe renta alguna (ni salario ni RB). Como se ha mencionado más arriba, la predisposición de un agente de este tipo puede sustentarse en una amplia gama de motivaciones como pueden ser una motivación autotélica hacia el trabajo, el deseo de obtener una mayor renta, o el seguimiento de una norma de tipo moral. Desde luego, cualquier trabajador podría, a priori, ajustarse a este perfil, debido a que cualquier trabajador podría albergar una norma moral que lo induzca a trabajar o podría desear disfrutar de una renta más elevada. No obstante, probablemente sean las profesiones de altos salarios las que alberguen un mayor número de LI; y muy especialmente colectivos como los profesionales liberales, los científicos, los artistas, los representantes políticos ${ }^{11} \mathrm{o}$ los deportistas sean los que mejor se ajusten a este

Cuadro 1. Orden de preferencias de LI

\begin{tabular}{ccc}
\hline \multicolumn{2}{c}{ Escenarios } & Utilidad ordinal \\
\hline Ego & Los demás & \\
$\mathrm{T}$ & $\mathrm{T}$ & 4 \\
$\mathrm{~T}$ & $\mathrm{NT}$ & 3 \\
$\mathrm{NT}$ & $\mathrm{T}$ & 2 \\
$\mathrm{NT}$ & $\mathrm{NT}$ & 1 \\
\hline
\end{tabular}


perfil, dadas las posibilidades de autorrealización y de obtención de reconocimiento social que ofrecen este tipo de profesiones.

Nótese que LI prefiere (T/NT) a (NT/T). Debido a que para LI el trabajo es un bien social, y no tanto una carga indeseable que hay que soportar para poder sobrevivir, esta se nos antoja una asunción razonable. No obstante, considerando que los juegos que se plantean en este trabajo son de $\mathrm{N}$ jugadores (ego y los demás), quizá la citada asunción sea poco plausible a partir de un determinado porcentaje de free-riders en la población, con el que podría parecer más razonable intercambiar el orden de la segunda y tercera preferencia. Esa modificación daría lugar a un nuevo tipo de agente (con un orden de preferencias característico de un juego del privilegiado). No obstante, se deja de lado aquí dicha complicación porque un agente de este tipo continuaría teniendo una estrategia dominante $\mathrm{T}$ y su conducta sería igual que la de LI, de modo que su inclusión sería redundante.

\section{Laborioso condicional (LC)}

En el caso anterior se mencionaba la posibilidad de que un agente laborioso pudiese llegar a alterar su orden de preferencias ante un elevado grado de free-riding en la población. La alteración del orden de preferencias que se ha barajado en ese caso carecía, como se ha visto, de relevancia teórica. No obstante, aún es posible plantearse una nueva variación de los supuestos que permita abordar un tema, ahora sí, muy relevante cuando se discute el impacto de una RB sobre los incentivos laborales: la condicionalidad de las disposiciones hacia el trabajo. LC es un tipo de agente que, si bien desea trabajar en primera instancia, no está dispuesto a hacerlo si el número de free-riders sobrepasa un determinado umbral. ${ }^{12} \mathrm{LC}$ no tiene NT/NT como la última preferencia y no cuenta con ninguna estrategia dominante. Concretamente, su orden de preferencias corresponde al de un dilema de la seguridad. Que LC no sitúe el escenario NT/NT en último lugar significa que este no depende de un salario o de una RB para subsistir. Este orden de preferencias puede corresponder con el de agentes que tienen asegurada la subsistencia porque disponen de recursos privados suficientes (porque son ricos, jóvenes que pueden vivir con sus padres, etc.) o porque viven en un país con un Estado de Bienestar lo suficientemente generoso.

Cuadro 2. Orden de preferencias de LC

\begin{tabular}{ccc}
\hline \multicolumn{2}{c}{ Escenarios } & Utilidad ordinal \\
\hline Ego & Los demás & \\
\hline $\mathrm{T}$ & $\mathrm{T}$ & 4 \\
$\mathrm{NT}$ & $\mathrm{T}$ & 3 \\
$\mathrm{NT}$ & $\mathrm{NT}$ & 2 \\
$\mathrm{~T}$ & $\mathrm{NT}$ & 1 \\
\hline
\end{tabular}


La decisión de LC de abandonar el trabajo cuando hay demasiados free-riders podría estar motivada sin duda por un deseo de sancionar la conducta de los mismos. No obstante, en el mundo real no parece demasiado plausible suponer que aquellos ciudadanos que desean sancionar a aquellos otros que se aprovechan de las prestaciones y servicios públicos sin contribuir a su provisión, abandonen sus empleos, sino que probablemente adopten otros cursos de acción que pueden ir desde (los más políticos como) votar a partidos que promuevan la eliminación o limitación de dichas prestaciones, hasta el fraude fiscal. Así pues, resulta más plausible suponer que la conducta de LC responde más bien a un cálculo de costes y beneficios: abandona el empleo cuando los beneficios derivados de la actividad laboral (salario, realización, etc.) no compensan los costes que esta implica. ${ }^{13}$

\section{Holgazán condicional (HC)}

En este caso entra en escena un agente que, a diferencia de los anteriores, no alberga disposiciones favorables al trabajo (de ahí la denominación de "holgazán"), pero que no tiene NT como estrategia dominante, de modo que su última preferencia corresponde a la situación NT/NT. Su orden de preferencias corresponde al del jugador de un dilema del gallina.

$\mathrm{HC}$ es un tipo de agente para quien el trabajo no es una actividad realizadora ni hacia la que sienta ningún tipo de obligación, poco influenciable por la desestima que puedan mostrarle sus conciudadanos por "parasitar" el trabajo de los demás, y con unas necesidades de consumo modestas. Sin embargo necesita de algún tipo de ingresos para subsistir, de manera que está dispuesto a trabajar si no hay más remedio. En este sentido, el trabajo constituye para él, única y exclusivamente, un instrumento para conseguir renta. Desde luego, a priori cualquier trabajador podría ajustarse a este perfil, pero, desde nuestro punto de vista, resulta plausible suponer que quizá este tipo de agente sea relativamente importante entre el colectivo de trabajadores más precarios, que se ven obligados a aceptar empleos en malas condiciones y con escaso potencial de autorrealización.

La RB, al ofrecerle una renta suficiente para subsistir, le daría a este individuo la oportunidad real para abandonar el trabajo. Desde nuestro punto de vista, este perfil de agente captura bien el tipo de individuo que los críticos de la RB tienen en mente cuando sostienen que la implantación de la medida supondría que los ciudadanos dejasen de trabajar. Esto es, los críticos parecen suponer que todos los trabajadores son HC.

13 Vale la pena mencionar que el incremento impositivo que supondría la entrada en vigor de una RB no tendría necesariamente que suponer que los LC redujesen su dedicación laboral. Podría ser que, al contrario, quisiesen trabajar más horas. Como es bien sabido, un aumento impositivo puede producir dos efectos opuestos: puede que algunos individuos opten por trabajar menos (ya que el coste de oportunidad del ocio ha bajado), mientras que otros decidan trabajar más para compensar la pérdida de ingresos. Este tipo de efectos totalmente opuestos son habituales en el mundo social (véase Elster, 2007, cap. 2 para una discusión detallada). 
Cuadro 3. Orden de preferencias de HC

\begin{tabular}{ccc}
\hline \multicolumn{2}{c}{ Escenarios } & Utilidad ordinal \\
\hline Ego & Los demás & \\
\hline $\mathrm{NT}$ & $\mathrm{T}$ & 4 \\
$\mathrm{~T}$ & $\mathrm{~T}$ & 3 \\
$\mathrm{~T}$ & $\mathrm{NT}$ & 2 \\
$\mathrm{NT}$ & $\mathrm{NT}$ & 1 \\
\hline
\end{tabular}

\section{Holgazán incondicional (HI)}

Nuestro último tipo de agente, $\mathrm{HI}$, es un individuo que, como HC, no alberga disposiciones favorables al trabajo, pero que, a diferencia de aquel, tiene la subsistencia asegurada por algún otro medio. Existe una amplia variedad de perfiles concretos englobables dentro de esta categoría (muchos de los cuales también se daban en LC), desde un individuo adinerado o un rentista, hasta un delincuente, pasando por un joven estudiante que vive con sus padres, una ama de casa que depende del salario de su marido, o un individuo con unas necesidades de consumo modestas y ciudadano de un país con un Estado de Bienestar generoso.

HI cuenta con una estrategia dominante NT y su orden de preferencias se ajusta al del jugador de un dilema del altruista, de modo que no trabajará bajo ninguna circunstancia.

Cuadro 4. Orden de preferencias de HI

\begin{tabular}{ccc}
\hline \multicolumn{2}{c}{ Escenarios } & Utilidad ordinal \\
\hline Ego & Los demás & \\
\hline NT & $\mathrm{T}$ & 4 \\
$\mathrm{NT}$ & $\mathrm{NT}$ & 3 \\
$\mathrm{~T}$ & $\mathrm{~T}$ & 2 \\
$\mathrm{~T}$ & $\mathrm{NT}$ & 1 \\
\hline
\end{tabular}

\section{Interacciones entre los agentes}

Los cuadros siguientes muestran los juegos que resultan cuando se pone a interactuar a los agentes. Como se explicitó más arriba, los juegos son de $\mathrm{N}$ jugadores, de manera que el jugador fila representa a ego mientras que el jugador columna representa a los demás. Los equilibrios se destacan en negrita cuando son óptimos. El único equilibrio subóptimo aparece en cursiva. Finalmente, pese a que esta información ya se ofrecía más arriba, como recordatorio, las estrategias dominantes se destacan subrayándolas. Primero se muestran los juegos simétricos y posteriormente los asimétricos. Como ya podía deducirse de la información ofrecida en el subapartado anterior, algunos de los 
juegos simétricos constituyen juegos clásicos de acción colectiva. Concretamente, la interacción entre LC y LC da lugar a un dilema de la seguridad, la de HC con HC a un dilema del gallina y la de $\mathrm{HI}$ con $\mathrm{HI}$ a uno del altruista.

\section{Juegos simétricos}

Cuadro 5. Juegos simétricos

\begin{tabular}{|c|c|c|c|c|c|}
\hline & \multicolumn{2}{|c|}{ LI vs. LI } & & \multicolumn{2}{|c|}{$L C$ vs. $L C$} \\
\hline & $\underline{T}$ & NT & & $\mathrm{T}$ & NT \\
\hline$\underline{T}$ & 4,4 & 3,2 & $\underline{T}$ & 4,4 & 1,3 \\
\hline \multirow[t]{3}{*}{ NT } & 2,3 & 1,1 & NT & 3,1 & 2,2 \\
\hline & \multicolumn{2}{|c|}{ HC vs. HC } & & \multicolumn{2}{|c|}{ HI vs. HI } \\
\hline & $\mathrm{T}$ & NT & & $\mathrm{T}$ & NT \\
\hline $\mathrm{T}$ & 3,3 & 2,4 & $\underline{T}$ & 2,2 & 1,4 \\
\hline NT & 4,2 & 1,1 & NT & 4,1 & 3,3 \\
\hline
\end{tabular}

\section{Juegos asimétricos}

Cuadro 6. Juegos asimétricos

\begin{tabular}{|c|c|c|c|c|c|}
\hline & \multicolumn{2}{|c|}{ LI vs. LC } & & \multicolumn{2}{|c|}{ LI vs. HC } \\
\hline & $\mathrm{T}$ & NT & & $T$ & NT \\
\hline$\underline{T}$ & 4,4 & 3,3 & $\underline{T}$ & 4,3 & 3,4 \\
\hline \multirow[t]{3}{*}{ NT } & 2,1 & 1,2 & NT & 2,2 & 1,1 \\
\hline & \multicolumn{2}{|c|}{ LI vs. HI } & & \multicolumn{2}{|c|}{ LC vs. HC } \\
\hline & $\mathrm{T}$ & NT & & $T$ & NT \\
\hline$\underline{T}$ & 4,2 & 3,4 & $\mathrm{~T}$ & 4,3 & 1,4 \\
\hline \multirow[t]{3}{*}{ NT } & 2,1 & 1,3 & NT & 3,2 & 2,1 \\
\hline & \multicolumn{2}{|c|}{ LC vs. HI } & & \multicolumn{2}{|c|}{ HC vs. HI } \\
\hline & $\mathrm{T}$ & NT & & $\mathrm{T}$ & $\underline{\text { NT }}$ \\
\hline $\mathrm{T}$ & 4,2 & 1,4 & $\mathrm{~T}$ & 3,2 & 2,4 \\
\hline NT & 3,1 & 2,3 & NT & 4,1 & 1,3 \\
\hline
\end{tabular}

Puede verse cómo siempre que una población está compuesta exclusivamente por agentes laboriosos el equilibrio resultante es $\mathrm{T} / \mathrm{T}$. Dicho equilibrio coincide con la primera 
preferencia de ambos jugadores, de modo que en esos escenarios no se da parasitismo ni explotación y no existe riesgo de evacuación del mercado de trabajo. En este sentido, no existe dilema de acción colectiva.

El único escenario que podría llegar a plantear problemas en este sentido es aquel en que la población está compuesta exclusivamente por LC. La matriz resultante corresponde con un dilema de la seguridad. Hay dos equilibrios, la cooperación o la defección mutua. El equilibrio de defección mutua es subóptimo respecto del de cooperación mutua. El problema en este caso es de confianza, los agentes solo cooperarán si tienen alguna garantía de que los demás lo harán. No obstante, en el caso que se plantea, en que ambos jugadores tienen disposiciones favorables al trabajo y suponiendo que las relaciones se perpetúan en el tiempo, resulta muy plausible suponer que la cooperación mutua acabe por convertirse en un equilibrio estable.

Si optamos por modelizar el escenario con un juego secuencial (como el de la Figura 1), en lugar de simultáneo, entonces sí que los agentes alcanzan el equilibrio óptimo. Es lógico que esto sea así porque, como acaba de mencionarse, el problema era de confianza. Cuando LC tiene conocimiento de que los demás agentes están cooperando, él opta por cooperar también.

Figura 1. Juego secuencial entre LC y LC

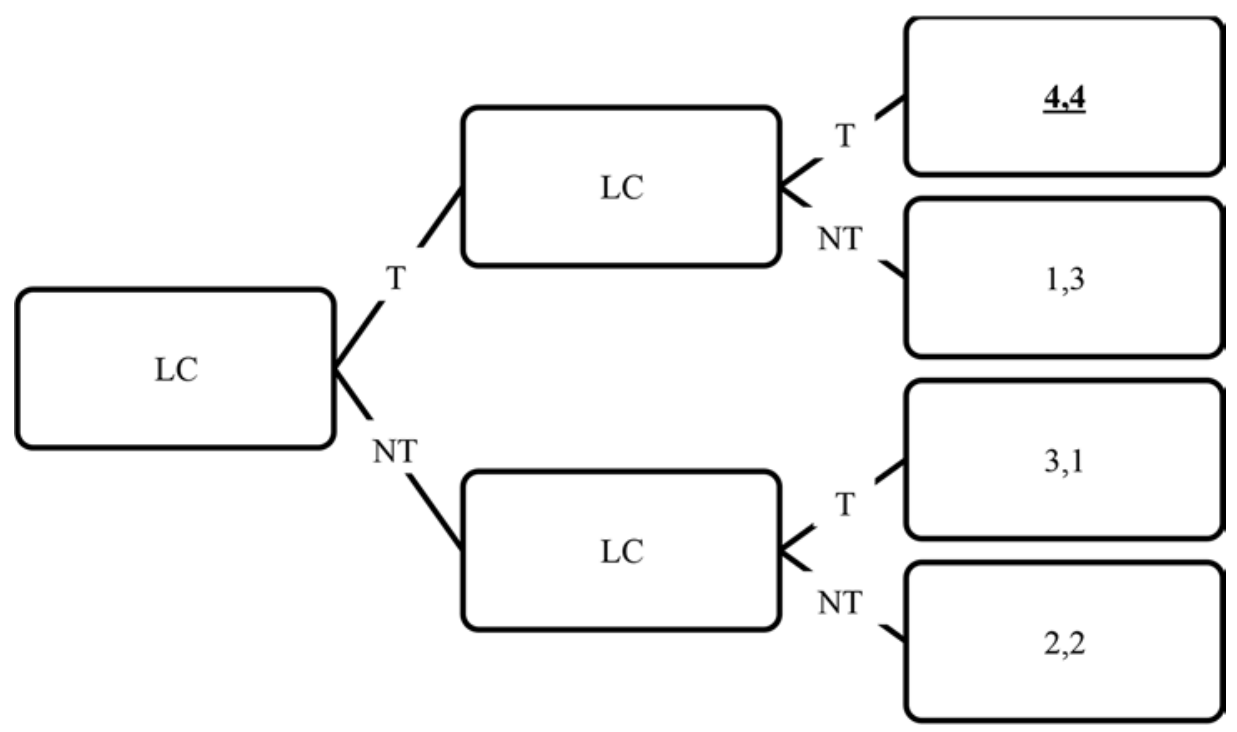

Sin embargo, resulta poco plausible suponer que en el mundo real todos los ciudadanos vayan a ser laboriosos, de manera que los juegos más interesantes son aquellos que plantean interacciones entre laboriosos y holgazanes.

Siempre que los LI interactúan con holgazanes (sean HC o HI), acaban trabajando en solitario. No obstante, nótese que en todos los casos el bien público se provee (aunque 
no al nivel máximo posible) y que el equilibrio es un óptimo de Pareto. Al fin y al cabo, en todos estos escenarios todos los agentes hacen lo que desean hacer: los holgazanes viven una vida ociosa (aunque en general austera) y los laboriosos trabajan tanto como quieren obteniendo así estima de sus conciudadanos, autoestima y autorrealización, una renta más alta que les permite un mayor nivel de consumo, etcétera.

Los escenarios que resultan de la interacción son bastante diferentes, empero cuando quienes entran en juego son los LC y no los LI. Cuando LC interactúa con HI, como puede verse, el equilibrio (NT/NT), pese a ser óptimo en términos matemáticos, dibuja un escenario ciertamente apocalíptico en términos sociales, ya que se produce una evacuación masiva del mercado de trabajo.

Por otra parte, la interacción entre LC y HC constituye un juego sin equilibrio, siendo por tanto imposible predecir lo que sucederá en dicho escenario (en términos de teoría de juegos es indeterminado).

En este caso, si optamos por modelizar el escenario a partir de juegos secuenciales se produce un resultado interesante. Como se puede ver en la Figura 2, el equilibrio varía en función de qué agente decida primero. Cuando empieza jugando LC, el equilibrio resultante es NT/T, que es subóptimo respecto del escenario T/T. En cambio, cuando empieza jugando HC se alcanza el equilibrio óptimo T/T. Así pues, el juego secuencial nos permite ser razonablemente optimistas respecto de la viabilidad de la RB en un mundo poblado por LC y HC. Como acabamos de ver, en el peor de los casos, el bien público se provee aunque no sea al máximo nivel posible. Pero, además, si tenemos en cuenta que en un mercado de trabajo real las interacciones son iteradas (esto es, los agentes no deciden una sola vez y para siempre si buscan o no un empleo, sino que la interacción se perpetúa en el tiempo), es de esperar que los agentes serán capaces de alcanzar el equilibrio óptimo.

Hasta ahora se han evaluado los hipotéticos escenarios que podrían producirse en un mundo formado exclusivamente por laboriosos, y en otro mundo, más plausible, integrado tanto por laboriosos como por holgazanes. Faltaría, por tanto, evaluar los posibles escenarios derivados de la aplicación de una RB en un mundo de holgazanes. A priori, si la posibilidad de encontrar un mundo poblado exclusivamente por laboriosos nos parecía improbable, lo mismo debería suceder con la opuesta y, de hecho, a nosotros nos lo parece así. No obstante, este no parece ser un escenario descartable para los críticos de la RB que alertan del peligro de una evacuación del mercado de trabajo si la medida es implementada. Así pues, veamos con detalle estos escenarios.

Vemos cómo, en dos de los tres escenarios, que son además los dos más plausibles, continúa habiendo agentes que optan por trabajar, de manera que el bien público es provisto (aunque ciertamente no al nivel máximo posible). No obstante, como ya parecía intuitivamente obvio, en un mundo poblado, única y exclusivamente por $\mathrm{HI}$, nadie trabajaría.

En el caso de un mundo poblado exclusivamente por HC, podemos ver que el juego resultante es un dilema del gallina. Como es bien sabido, en este juego "hay cooperación 
Figura 2. Juegos secuenciales entre LC y HC
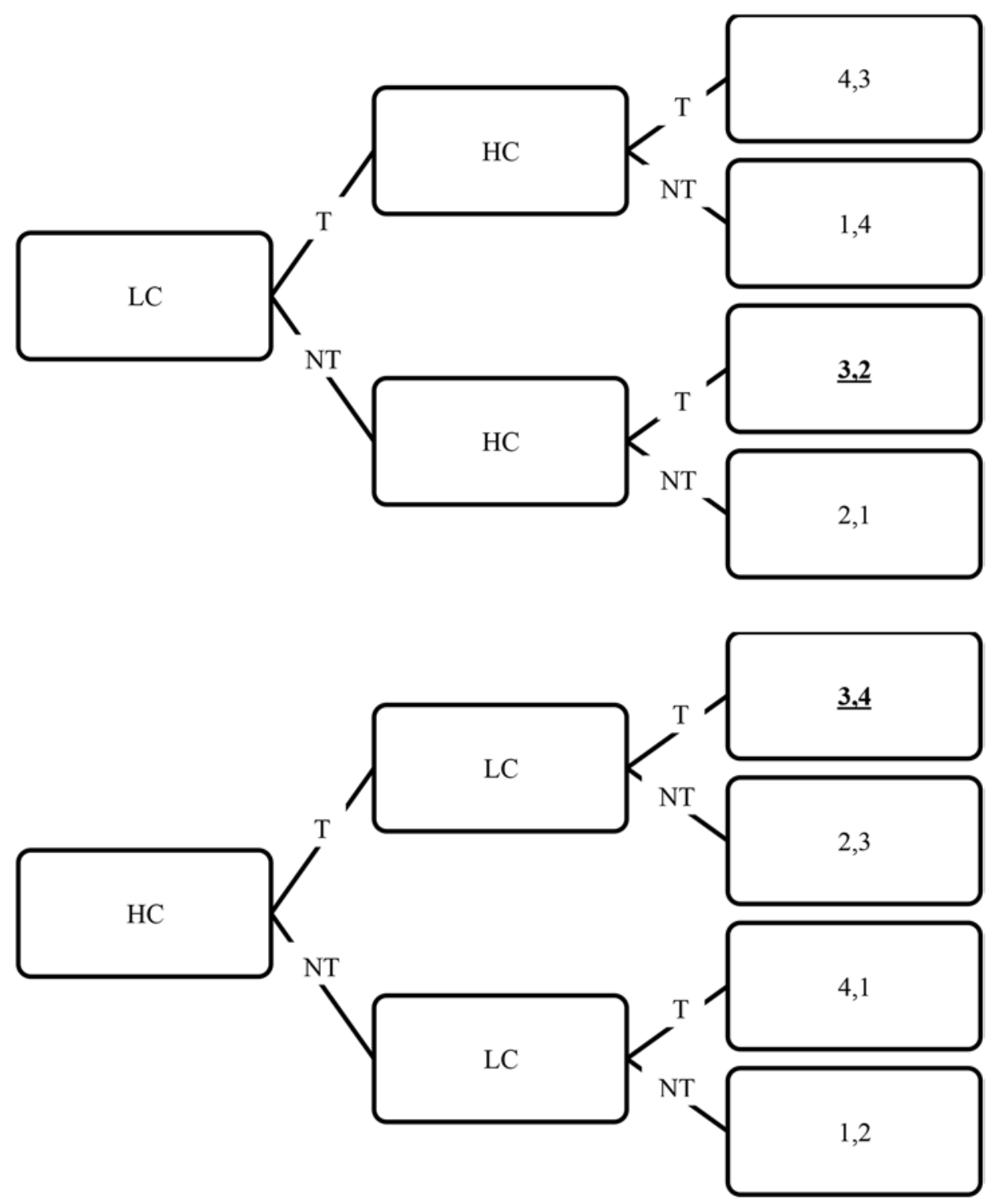

parcial (un jugador coopera y el otro no), lo que conduce al problema de quién hará el trabajo por los dos (en juegos de $\mathrm{N}$ jugadores, por tanto, la distribución del trabajo será asimétrica)" (Linares, 2007: 53). Pero, en cualquier caso, siempre habrá agentes (los gallinas) que trabajen de modo que la provisión del bien público está asegurada.

En este caso, obviamente, la modelización del escenario en forma de juego secuencial confirma este resultado. 
Figura 3. Juego secuencial entre $\mathrm{HC}$ y HC

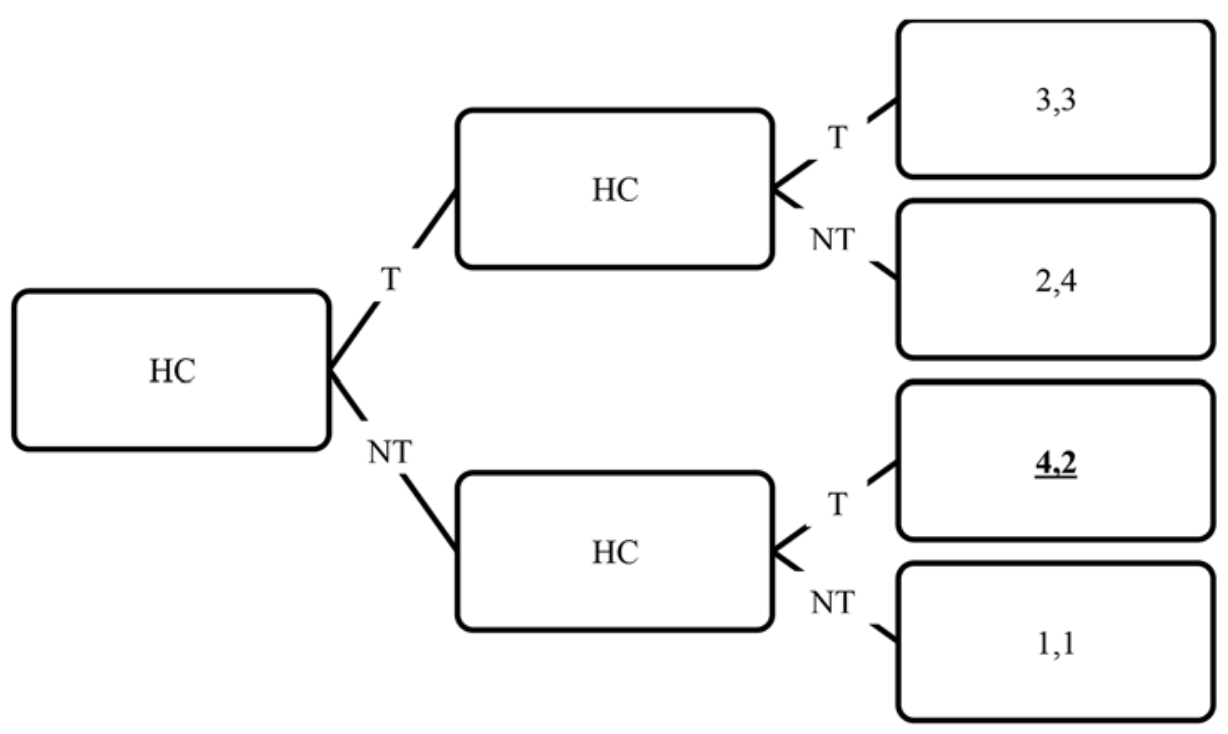

Finalmente, en un mundo poblado por $\mathrm{HC}$ y $\mathrm{HI}$, los primeros serían los únicos en trabajar, de manera que serían explotados por los segundos. No obstante, una vez más, es preciso notar que, incluso en este desconfortante escenario, el bien público sería provisto (aunque no al máximo posible) y el equilibrio alcanzado constituiría un óptimo de Pareto.

\section{DISCUSIÓN Y CONCLUSIONES}

En la sección anterior hemos visto cómo en al menos 7 de los 10 escenarios planteados no se produciría evacuación del mercado de trabajo y la RB sería viable con una cuantía mayor o menor dependiendo del caso concreto. No obstante, tendríamos 2 escenarios en que sí se da evacuación y el bien público no se produce, y aún un último escenario en el que no sabemos qué podría llegar a suceder (aunque la modelización del mismo a partir de un juego secuencial permite prever que en ese escenario la RB sería provista al máximo nivel posible).

Desde luego, sería absolutamente inadecuado concluir este trabajo sosteniendo que existe una elevada probabilidad de que la RB fuese viable y resultase sostenible en el tiempo. En cambio, es preciso valorar la plausibilidad de cada uno de los escenarios planteados a fin de poder ofrecer una conclusión más informada. En este sentido, desde nuestro punto de vista, aunque esta es una cuestión que evidentemente debería dilucidarse empíricamente, parece plausible suponer que el tipo de agentes más importantes en términos cuantitativos en las sociedades como las nuestras probablemente sean los LI $\mathrm{y}$, en menor medida, los $\mathrm{H}$ de uno $\mathrm{u}$ otro tipo. Visto que tanto en aquellos escenarios en los que toda la población está constituida por LI como, de manera más relevante, en aquellos en que los LI interactúan con otros agentes, no se produce problema alguno de evacuación del mercado de trabajo y el bien público, la RB se produce en mayor o 
menor medida, creemos que la conclusión de este trabajo debe ser optimista respecto de la viabilidad de la propuesta.

Empezaremos la discusión refiriéndonos a LC. Como se dijo, LC es un agente que alberga una disposición favorable al trabajo, pero que en un momento dado podría permitirse vivir sin trabajar, ya sea merced a los recursos privados (es rico, un joven que puede vivir con sus padres, etc.) ya sea porque vive en un país con un Estado de Bienestar amplio y generoso. Por esta razón, desde nuestro punto de vista, LC es un tipo de agente poco relevante en términos cuantitativos. Si LC tiene realmente disposiciones favorables hacia el trabajo, aunque estas sean condicionales, en principio, no debería abandonarlo porque se aplique una RB. De todos los motivos que se enumeraron para sostener una tal disposición: sentido del deber (seguimiento de una norma), autorrealización, búsqueda de reconocimiento social, deseo de ingresar una renta mayor, etc.; la mayoría de ellos no deberían verse socavados (o no totalmente) por la introducción de una RB. Tan solo el sentido del deber podría verse erosionado en un escenario en el que existiese un gran número de individuos holgazanes. Lo que quizá sí que podría hacer LC si viviese en una sociedad con RB sería trabajar menos horas (ya que una parte de su renta está asegurada por la RB) y dedicar parte de su tiempo a otras actividades. Pero esto, lejos de ser un problema, es uno de los beneficios que los defensores de la medida esgrimen con más fuerza, en tanto en cuanto esta podría ser una buena herramienta para promover un mayor reparto del empleo, ${ }^{14}$ un mayor reparto del trabajo doméstico, 15 favorecer el voluntariado y la participación ciudadana, ${ }^{16}$ la formación, el autoempleo ${ }^{17}$ y un largo etcétera.

En definitiva, si LC dejase de trabajar totalmente como consecuencia de la introducción de una $\mathrm{RB}$, esto probablemente sería porque en realidad no estaríamos ante un L, sino ante un $\mathrm{H}$ de alguno de los dos tipos. En cambio, existen buenas razones para pensar que un agente realmente laborioso no abandonaría el mercado de trabajo porque se introdujese una RB al nivel de subsistencia.

Centrémonos ahora el caso de los HI. Sin duda, en las sociedades realmente existentes hay ciudadanos de este tipo. No obstante, si se evalúa la relevancia de los mismos en términos cuantitativos podemos ver que no tenemos demasiados elementos para la preocupación. Como se dijo, HI podría responder a diversos perfiles, por ejemplo a) al de un ciudadano rico (un rentista), b) al de un joven que cuenta con alguien que sufraga sus gastos por él, c) al de un ama de casa o d) al de un ciudadano de un país con un Estado del Bienestar muy generoso.

14 Si la gente que actualmente trabaja decidiese trabajar menos horas, esas horas quedarían libres para toda la gente que actualmente se encuentra desempleada. En este sentido, nótese que, quizá sorprendentemente, la introducción de una RB podría incluso comportar un aumento del número global de horas trabajadas (siempre y cuando creciese la oferta de empleo). Véase Noguera (2002a, 2002b, 2003, 2004, 2005), Standing (1986, 1988, 1992, 2002), Van Parijs (1997), Van Parijs y Vanderborght (2006) o Van Parijs et al. (2002).

15 Véase, por ejemplo, Jordan (1992), Standing (1986) o Schroeder (2001).

16 Véase Noguera (2002a, 2003, 2004, 2005), Van Parijs (1997), Van Parijs y Vanderborght (2006), Van Parijs et al. (2002) o Wright (2000).

17 Véase Noguera $(2005,2009)$. 
Discutamos estos casos particulares uno por uno. Por un lado, evidentemente, la RB no alteraría la conducta laboral de los ricos rentistas que continuarían pudiendo disfrutar de su estilo de vida exactamente del mismo modo que lo hacen ahora. No obstante, por motivos obvios, el porcentaje de este tipo de individuos en la población no puede ser demasiado alto y, por tanto, no plantean ningún problema de viabilidad para la RB. Pero además, es preciso notar que, en términos normativos, la RB posibilita en este caso un escenario preferible al actual. En las sociedades actuales existen ya ciudadanos rentistas que no trabajan y una gran masa de no rentistas que sí lo hacen. Si se introdujese una $\mathrm{RB}$, toda la población no rentista continuaría trabajando, pero al menos ahora cobrarían una RB, cosa que los situaría en mejor situación que la actual respecto de los rentistas.

En el caso de los jóvenes que no trabajan porque están estudiando y tienen una familia que sufraga sus gastos, es vital argumentar que esta situación no solo no tiene nada de indeseable (ni en términos normativos ni en ningún otro), sino todo lo contrario. Además, la RB extendería ese derecho real a continuar estudiando y no tener que incorporarse tan pronto al mercado de trabajo también a aquellos otros jóvenes que no cuentan con unos padres que puedan costear sus estudios.

En tercer lugar, en lo que se refiere a las amas de casa que no toman parte en el mercado de trabajo porque se ocupan del trabajo doméstico, quizá podría suceder que la introducción de una RB supusiese la perpetuación de estas mujeres en dicha situación e incluso que algunas de ellas que se encuentran ocupadas en empleos de media jornada se retirasen definitivamente del mercado de trabajo. No obstante, aunque esto fuese así, es importante destacar que no existiría nada normativamente indeseable en dicho escenario. Nótese que el problema de que algunas mujeres no puedan incorporarse plenamente al empleo porque deben hacer frente en solitario al trabajo reproductivo no es que el trabajo remunerado sea mejor en ningún sentido que el trabajo doméstico. En realidad el problema radica en que esas mujeres no puedan decidir libremente si desean o no incorporarse al mercado de trabajo y que al quedar excluidas del empleo pasan a depender económicamente de sus maridos. Nótese que, en este sentido, la introducción de una RB sería netamente beneficiosa para las mujeres, ya que aumentaría sus oportunidades y su poder de negociación frente a sus parejas. En palabras de Van Parijs (2002) "I've always found it crucial to distinguish between measures that modify women's choices by restricting their options and by expanding their options. Basic Income is unambiguously of the latter kind, and (...) I find that there is something insulting about considering women, in particular less-skilled women, to be less able than men to make a wise use of these expanded options".

Por otro lado, se destaca que, de hecho, resulta mucho más plausible suponer que la implementación de una RB tendría el efecto contrario y que, al favorecer -como se ha señalado más arriba- el reparto del empleo y del trabajo doméstico, facilitaría la incorporación de las mujeres al trabajo productivo. ${ }^{18}$

18 Acerca de la posible incidencia de una RB sobre los incentivos laborales de las mujeres (y sobre su permanencia o no en el ámbito doméstico), véase Jordan (1992), Noguera (2002a, 2003, 2004, 2005), Pinilla (2004, 2006), 
Finalmente, aún falta discutir el caso más complejo, el del individuo con unas necesidades de consumo modestas, ciudadano de un país con un Estado del Bienestar generoso. Parece claro que este es el tipo de sujeto y de situación que tienen en mente los críticos de la RB cuando alertan del riesgo de evacuación del mercado de trabajo y de acabar construyendo una sociedad de parásitos. Nótese que HC también plantea esta amenaza, ya que como HI no alberga disposiciones favorables hacia el trabajo. La diferencia entre ambos, en este caso particular, viene dada por el contexto institucional en que se encuentran: HC no disfruta de un Estado del Bienestar generoso que pueda permitirle el lujo de vivir sin trabajar.

En este sentido, la conclusión del argumento en términos de política social suele ser que es necesario evitar aquellas políticas pasivas que no fuerzan al perceptor a buscar empleo de forma activa. Esta crítica se esgrime habitualmente contra políticas como los subsidios de desempleo o las rentas mínimas de inserción (RMI) a las que se acusa de dar lugar al fraude y a las denominadas trampas de la pobreza y del paro. El problema básico con estas trampas consiste en el hecho de que una persona que está cobrando una prestación de este tipo tiene un notorio desincentivo para aceptar una oferta de empleo, ya que al hacerlo pierde (ya sea total o parcialmente) la prestación. Para eliminar el efecto de la trampa, el salario del nuevo empleo tiene que superar ampliamente la cuantía de la prestación, cosa que no suele suceder, ya que los empleos a los que optan los beneficiarios de estos subsidios suelen ser precarios.

Si bien este problema existe y está muy presente en el debate acerca del diseño de las políticas sociales, también es cierto que se trata de un problema que, a priori, no parece que debiera preocupar demasiado a los defensores de la RB. Hechos como que el número de perceptores de rentas mínimas sea en general muy escaso y se trate además frecuentemente de personas con dificultades de inserción social, permiten a nuestro juicio ser optimistas respecto de la viabilidad de la RB. Si la gran mayoría de trabajadores no se conforma con llevar una vida ociosa y cobrar una RMI o una prestación asistencial (cuya cuantía cae a menudo por debajo del umbral de la pobreza), no hay ninguna razón para pensar que en cambio sí que abandonarían sus empleos si se introdujese una RB que alcanzase para la subsistencia material. ${ }^{19}$

En este punto vale la pena discutir explícitamente la posible incidencia de una RB sobre la conducta laboral de un perfil de trabajador que se ha sostenido que podría tener una presencia importante entre los HC (aunque este vuelve a ser un supuesto que habría que contrastar empíricamente): los trabajadores precarios que realizan empleos en malas condiciones, de bajos salarios y con poco potencia de autorrealización. Por un lado, una de las principales justificaciones de la RB consiste en el hecho de que esta ofrecería a dichos trabajadores la oportunidad real de abandonar sus empleos (una opción salida).

Robeyns (2000), Standing (1986, 1988, 1992, 2002), Van Parijs (1997), Van Parijs y Vanderborght (2006), Van Parijs et al (2002), Wright (2000).

19 Vale la pena notar que la RB, además, puede ser una buena medida de activación de la fuerza de trabajo y de reducción del fraude, ya que resuelve las trampas anteriormente discutidas (Groot y Van der Veen 2002; Jordan, 1992; Noguera, 2002a, 2002b, 2002c, 2003, 2004, 2005; Schroeder, 2001; Standing, 1986, 1988, 1992, 2002; Van Parijs, 1992, 1997; Van Parijs y Vanderborght, 2006). 
Sin embargo, obviamente, si todos los trabajadores que en la actualidad realizan esos trabajos abandonasen sus empleos, el resultado podría ser catastrófico. Creemos que existe una razón adicional para ser optimistas además de la que acaba de mencionarse. Por un lado, la RB aumentaría el poder de negociación (individual y colectivo) de esos trabajadores (les ofrecería una opción voz), que podrían reclamar unas mejores condiciones laborales, mejores salarios, etc. Por otro lado, si, como consecuencia de la introducción de una RB se empezase a producir un detraimiento de la oferta de trabajo en esos segmentos del mercado, los empresarios se verían igualmente obligados a mejorar las condiciones de los mismos. Por ambas vías, por tanto, parece razonable suponer que la introducción de una RB, más que un abandono masivo del mercado de trabajo por parte de los trabajadores precarios, podría suponer una mejora de esos empleos.

En definitiva, aunque esta es una cuestión que habría que dilucidar de manera empírica, parecería que resulta razonable sugerir que buena parte de los ciudadanos se ajusta a un perfil LI y que, por tanto, la introducción de una RB resulta una propuesta viable que no produciría una evacuación masiva del mercado de trabajo. Tampoco resulta plausible suponer que absolutamente toda la población vaya a estar compuesta por LI y, sin duda, en cualquier sociedad realmente existente habrá, como se ha dicho, un determinado número de $\mathrm{H}$. Como hemos visto, en todos esos escenarios el equilibrio resultante sería óptimo desde un punto de vista matemático, pero vale la pena notar que también lo sería desde un punto de vista "social" si se acepta el argumento de Van Parijs (1997) de que no existe ningún motivo para considerar que una disposición laboriosa sea éticamente mejor que una centrada en lo necesario para la subsistencia, de modo que la RB abriría la puerta a una sociedad con una mayor libertad real en el sentido de que todo el mundo podría escoger libremente una forma e intensidad de dedicación laboral más acorde con sus preferencias.

No quisiéramos finalizar este trabajo sin indicar algunos posibles desarrollos futuros del mismo. Un primer paso a dar puede consistir en una calibración empírica más sistemática de los supuestos del modelo, por ejemplo, a partir de datos de encuesta o experimentales, que permitan discernir cuáles de los anteriores escenarios son más probables y realistas. Una segunda línea de trabajo, estrechamente ligada con la anterior, debe pasar por la exploración de escenarios de interacción más complejos en los que intervienen varios tipos de agentes de manera prolongada en el tiempo. En este sentido, la utilización de herramientas como la simulación multiagente, muy novedosas en ámbitos como las ciencias sociales y la filosofía, ofrece nuevas oportunidades poco costosas y relativamente sencillas para la exploración de escenarios de interacción complejos mediante modelos formales. ${ }^{20}$ 


\section{REFERENCIAS}

Elster, Jon. 1986. "Comment on Robert J. van der Veen and Philippe van Parijs". Theory and Society 15 (5): 709-721.

2007. Explaining Social Behavior. More Nuts and Bolts for the Social Sciences. Cambridge: Cambridge University Press.

González-Bailón, Sandra (ed.). 2011. “Labour Market Behaviour and Unconditional Benefits: An AgentBased Model". II Encuentro del Grupo de Sociología Analítica de la Federación Española de Sociología. Sevilla (España).

Groot, Loek y Van der Veen, Robert. 2002. “¿Cuán Atractivo Resulta el Ingreso Básico para los Estados de Bienestar Europeos?". En La Renta Básica en la Agenda: Objetivos y Posibilidades del Ingreso Ciudadano, editado por R. van der Veen et al. Buenos Aires, Madrid: Miño y Dávila: Ciepp, 21-66.

Heckathorn, Douglas. 1996. "The Dynamics and Dilemmas of Collective Action". American Economic Review 6: 250-277.

Jordan, Bill. 1992. "Basic Income and the Commond Good". En Arguing for Basic Income. Ethical Foundations for a Radical Reform, editado por P. van Parijs. Londres: Verso, 155-177.

Linares, Francisco. 2007. “El Problema de la Emergencia de Normas Sociales en la Acción Colectiva. Una Aproximación Analítica". Revista Internacional de Sociología LXV (46): 131-160.

Noguera, José A. 2002a. "¿Renta Básica o Trabajo Básico? Algunos Argumentos desde la Teoría Social”. Sistema 166: 61-85.

. 2002b. "Renta Básica y Crisis del Estado del Bienestar". El Vuelo del Ícaro. Revista de Derechos Humanos, Crítica Política y Análisis de la Economía 2/3: 109-126.

. 2002c. "A favor de la Renda Bàsica: per un Debat Rigorós i Obert. Comentaris sobre La Renda Bàsica a Catalunya, de José Iglesias Fernández". Nous Horitzons 167: 44-49.

2003. "Seguridad de la Cuna a la Tumba. La Renta Básica como Renovación del Estado del Bienestar". Jornada de Debate "Renda Bàsica". Xarxa Maragall-Fundació Rafael Campalans.

2004. "Citizens or Workers? Basic Income vs. Welfare-to-Work Policies". Journal of Law and Urban Studies 2 (1): 103-124.

2005. "Renda Bàsica, Mercat de Treball i Incentius Laborals". Revista del Consell de Treball Econòmic i Social de Catalunya.

2009. “Renta Básica, Política de Rentas e Incentivos Laborales". En Desigualdades en las Sociedades Contemporáneas, editado por H. Ganzeboom P. Taylor-Gooby, J. Roemer, A. Villar y J. A. Noguera. Sevilla: Centro de Estudios Andaluces-Junta de Andalucía.

Noguera, José A. y de Wispelaere, Jurgen. 2006. "A Plea for the Use of Laboratory Experiments in Basic Income Research". Basic Income Studies 1 (2): 1-8.

Pinilla, Rafael. 2004. La Renta Básica de Ciudadanía. Una Propuesta Clave para la Renovación del Estado del Bienestar. Barcelona: Icaria.

2006. Más allá del Bienestar. La Renta Básica de la Ciudadanía como Innovación Social Basada en la Evidencia. Barcelona: Icaria.

Robeyns, Ingrid. 2000. "Hush money or emancipation fee? A gender analysis of Basic Income". En Basic Income on the agenda: policy objectives and political chances, editado por R. Van der Veen y L. Groot. Amsterdam: Amsterdam University Press, 121-136.

Schroeder, Doris. 2001. “Wickedness, Idleness and Basic Income”. Res Publica 7: 1-12.

Standing, Guy. 1986. Unemployment and Labour Market Flexibility: The United Kingdom. Geneva: International Labour Office.

1988. Unemployment and Labour Market Flexibility: Sweden. Geneva: International Labour Office. . 1992. "The Need for a New Social Consensus". En Arguing for Basic Income. Ethical Foundations for a Radical Reform, editado por P. van Parijs. Londres: Verso, 47-60.

2002. Beyond the New Paternalism. Basic Security as Equality. London: Verso.

Steiner, Hillel. 1992. "Three just taxes". En Arguing for Basic Income. Ethical Foundations for a Radical Reform, editado por P. van Parijs. Londres: Verso, 81-92. 
Van Parijs, Philippe. 1992. "The Second Marriage of Justice and Efficiency". En Arguing for Basic Income. Ethical Foundations for a Radical Reform, editado por P. van Parijs. Londres: Verso, 215-240. 1997. Real Freedom for All. What (if Anything) Can Justify Capitalism? Oxford: Oxford University Press. 2002. "Interview with Pascal Couillard". USBIG Newsletter 3 (17). En: http://www.usbig.net/ newsletters/17AUG-SEP2002.htm

Van Parijs, Philippe y Vanderborght, Yannick. 2006. La Renta Básica. Una Medida Eficaz para Luchar contra la Pobreza. Barcelona: Paidós.

Van Parijs, Philippe (ed.). 2002. “El Ingreso Básico y sus Parientes: el Ingreso Básico Parcial versus el Earned Income Tax Credit (EITC) y la Reducción de los Aportes a la Seguridad Social, como Formas Alternativas de Enfrentar la Nueva Cuestión Social". En La Renta Básica en la Agenda: Objetivos y Posibilidades del Ingreso Ciudadano, editado por L. Groot et al. Buenos Aires, Madrid: Miño y Dávila: Ciepp, 89-141.

Widerquist, Karl. 2005. "A Failure to Communicate: What (If Anything) Can We Learn From the Negative Income Tax Experiments". Journal of Socio-Economics 34: 49-81.

Widerquist, Karl (ed). 2013. Basic Income: An Anthology of Contemporary Research. Oxford: Wiley- Blackwell. Wright, Erik O. 2000. "Propuestas Utópicas Reales para Reducir la Desigualdad de Ingresos y Riqueza". En Razones para el Socialismo, editado por Roberto Gargarella et al. Barcelona, Buenos Aires, México: Paidós, 195-221.

Jordi Tena-Sánchez es profesor agregado en el Departamento de Sociología de la Universitat Autònoma de Barcelona y miembro del Grupo de Sociología Analítica y Diseño Institucional (GSADI). Sus intereses académicos giran en torno a la aplicación de los principios de la teoría social analítica al estudio de diversos aspectos de las sociedades contemporáneas. E-mail: jordi.tena@uab.cat.

José A. Noguera es profesor titular de sociología en la Universitat Autònoma de Barcelona y director del grupo de investigación GSADI (Grupo de Sociología Analítica y Diseño Institucional). Sus actuales áreas de trabajo son la política social (en especial la renta básica universal), la sociología fiscal, la teoría sociológica y la simulación social. Es miembro del consejo de redacción de la revista Basic Income Studies, y del consejo consultivo internacional de la BIEN (Basic Income Earth Network). E-mail: jose.noguera@uab.cat. 